\title{
Study of micro particles deposition in ribbed channel: A numerical investigation in turbulent airflow
}

\author{
Kianoosh Samimi ${ }^{1,2}$, Helia Sharafi ${ }^{3}$, Essolé Padayodi ${ }^{4}$ And Seyed Amir Bahrani ${ }^{5,6, a}$ \\ 1 LGCGM, INSA de Rennes, 20 avenue des Buttes de Coësmes, 35708 Rennes France \\ 2 Imen Rah consulting Engineers. Research and development service, Tehran, Iran \\ 3 School of Civil Engineering, University of Shiraz, Shiraz, Iran \\ 4 IRTES-SeT, Université de Technologie de Belfort-Montbéliard, 90010 Belfort, France \\ 5 Université de Lorraine, LEMTA, UMR 7563, 54500 Vandoeuvre-lès-Nancy, France \\ ${ }^{6}$ CNRS, LEMTA, UMR 7563, 54500 Vandoeuvre-lès-Nancy, France
}

Received 19 July 2013, Accepted 8 February 2014

\begin{abstract}
The air is modeled as incompressible flow in a ribbed channel. The existence of ribs on channel surface causes the turbulence in flow, therefore we must solve the turbulence equations. There are previous experimentally investigations on turbulence flow in ribbed channels. In this article, the results of numerical simulation are compared with experimental results and the effectiveness of $k$ - $\varepsilon$ model in solving the problem of micro-particle deposition with Lagrangian model is studied because of complexities of turbulent flow, the problem is modeled both 2- and 3-dimensionally. After solving the fluid domain, the particle equations of motion are solved separately and finally the particle deposition on channel surface is calculated. Considering the physic and complications of turbulent flows, the results of 2-D simulation are different with experimental results, despite of 3-D results, which are in complete accordance with experimental results.
\end{abstract}

Key words: Particles deposition / ribbed channel / numerical simulation / turbulence $k-\varepsilon$ model.

\section{Introduction}

Aerosols transfer and deposition in turbulent channels are always important and widely used in engineering projects $[1-3]$. In the design of air conditioning systems, it's important to study the indoor air quality because people is mostly in the indoor areas. The indoor air comes from the out after passing through transforming channels. Considering the most human expense of their time in indoor areas, it is very important to check indoor air pollution for air conditioning projects. The air pollution mostly caused by aerosols smaller than $10 \mu \mathrm{m}$ [2] and aerosol concentration in the air vary locally. Epidemiological evidence suggests a positive correlation between outdoor $\mathrm{PM}_{10}$ (particular matter less than $10 \mu \mathrm{m}$ in diameter) concentrations and mortality in urban areas. Therefore it is desirable to remove these particles from air conditioning systems. The most common way is to equip the absorption filter at air flow inlet, obviously this method needs energy [4] and it is not very effective [5].

If aerosols deposition is augmented in ventilation channel, the effective filtration will be enhanced. The enhancement of small particle deposition along the length

\footnotetext{
${ }^{a}$ Corresponding author:

seyed-amir.bahrani@univ-lorraine.fr
}

of ventilation duct may present an effective alternative to filtration. The aerosol deposition augments by roughening the internal area of channel. Also, particle deposition can be increased by heat transfer. The later method is used in turbine blades and nuclear reactor cooling [4]. One way of roughened channel area is to add the ribs on channel surface.

Although there are a few investigations on numerical simulation of particle deposition in ribbed channels, the turbulent flow in ribbed channels is surveyed widely by researchers. In 2006, Liu and his colleagues used two turbulence models "LES" and "RANS" in order to simulate numerically the turbulent flow in electrical devices [6]. Also in the same year, Viswanthan and Tafti [7] modeled a turbulent flow in a channel which has ribs on both bed and ceiling of channel, they used a Detached Eddy Simulation "DES" model. They compared the numerical results with experimental data [8]. In 2007, Ahn et al. [9] modeled fluid flow with ribs on both bed and ceiling of channel by use of LES. They assumed that rotating channel is a model of cooling flow on turbine blades. Tian and Ahmadi [10] compared the results of different numerical turbulence models for a simple channel and confirmed the results of $k-\varepsilon$ model which is used by fluent software. They can show an acceptable prediction for particle deposition. 


\begin{tabular}{|c|c|}
\hline \multicolumn{2}{|c|}{ Nomenclature } \\
\hline$A$ & Area $\left(m^{2}\right)$ \\
\hline$C_{\infty}$ & Aerosol concentration (particle. $\mathrm{m}^{-3}$ ) \\
\hline$d_{\mathrm{p}}$ & Particle diameter $(\mathrm{m})$ \\
\hline$D_{e}$ & Hydraulic mean diameter $(\mathrm{m})$ \\
\hline$F_{i}^{\mathrm{L}}$ & Lifting force \\
\hline$f$ & Friction factor $(-)$ \\
\hline$g_{i}$ & Acceleration of gravity \\
\hline$H$ & Height of rib $(\mathrm{m})$ \\
\hline$J$ & Deposited aerosol flux (particle.m ${ }^{-2} \cdot \mathrm{s}^{-1}$ ) \\
\hline$k$ & Turbulence kinetic energy $\left(\mathrm{m}^{2} \cdot \mathrm{s}^{-2}\right)$ \\
\hline$\Delta L$ & Distance $(\mathrm{m})$ \\
\hline$M$ & Particle mass detected (g) \\
\hline$p$ & Static pressure $\left(\mathrm{m} . \mathrm{s}^{-1}\right)$ \\
\hline$P$ & Pitch length of ribbed surface (m) \\
\hline$\Delta P$ & Differential pressure loss $(\mathrm{Pa})$ \\
\hline$Q$ & $\begin{array}{l}\text { Volumetric flow rate through the air } \\
\text { filter samplers }\left(\mathrm{m}^{3}\right)\end{array}$ \\
\hline Re & Reynolds number $(-)$ \\
\hline$t$ & Sampling time $(\mathrm{s})$ \\
\hline$u_{i}$ & Phase velocity $\left(\mathrm{m} . \mathrm{s}^{-1}\right)$ \\
\hline$u_{i}^{\mathrm{p}}$ & Particle velocity $\left(\mathrm{m} \cdot \mathrm{s}^{-1}\right)$ \\
\hline$U^{2}$ & Bulk mean velocity $\left(\mathrm{m} . \mathrm{s}^{-1}\right)$ \\
\hline$V_{d}$ & Mass transfer coefficient $\left(\mathrm{m} \cdot \mathrm{s}^{-1}\right)$ \\
\hline$\varepsilon$ & Turbulence dissipation rate $\left(\mathrm{m}^{2} \cdot \mathrm{s}^{-3}\right)$ \\
\hline$\delta$ & Half of channel height $(\mathrm{m})$ \\
\hline$\mu$ & Fluid viscosity (Pa.s) \\
\hline$\mu_{\mathrm{t}}$ & Turbulent eddy viscosity (Pa.s) \\
\hline$\rho$ & Fluid density $\left(\mathrm{kg} \cdot \mathrm{m}^{-3}\right)$ \\
\hline$\rho_{\mathrm{p}}$ & Particle density $\left(\mathrm{kg} \cdot \mathrm{m}^{-3}\right)$ \\
\hline$\overline{\bar{\tau}}$ & Stress tensor $(\mathrm{Pa})$ \\
\hline
\end{tabular}

\section{Subscripts and superscripts \\ acetate acetate \\ filter air filter}

More investigations upon ribbed channels are carried out recently. In 2010, Ahn and Lee [11] worked on large eddy simulation (LES) of turbulent flow and heat transfer in a channel with both detached and attached rib array.

In recent investigation, by use of fluid domain solution the micro particle equation of motion is solved independently in fluent software. For solving fluid domain by fluent, the grid independent results for $k-\varepsilon$ model with different wall conditions are obtained. By use of a same channel geometry with Lai's experimental work, the results of numerical work are compared with experimental data. This comparison shows the numerical simulation capability for modeling aerosol filtering problems in ventilation channels.

\section{Physical model and solving method}

\subsection{Geometry of experimental model}

For physical simulation the geometry of Lai's experiment is used [1]. In Figure 1, the experimental apparatus and the ribs on its bed are shown. Channel cross section

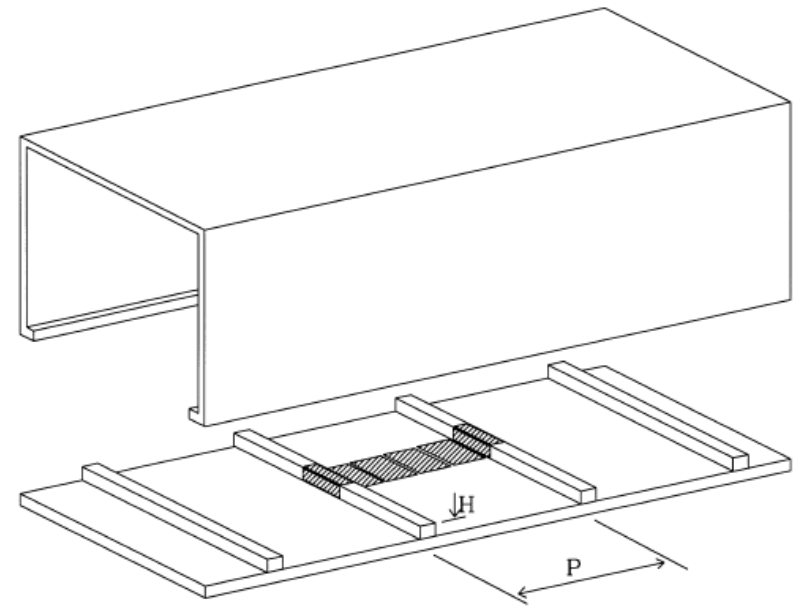

Fig. 1. Ribbed channel schematic. The dashed area is test section [1].

is $150 \mathrm{~mm} \times 150 \mathrm{~mm}$. The channel is consisted of ten galvanized sections. The length of each section is $500 \mathrm{~mm}$. Channel hydraulic diameter is $150 \mathrm{~mm}$. Each galvanized rib has $6 \mathrm{~mm}$ height $(H)$ and the pitch length $(P)$ between two successive ribs is $60 \mathrm{~mm}$.

The test section was located at the 10th galvanized channel. Thereby for the inlet flow to this area the flow is fully turbulent. As shown in Figure 1, the test section is placed in the middle of channel and particle deposition is measured in this section although the particle deposition in depth is neglected. Hence, for flow simulation, first a 2-dimensional model is used. Despite of the simplicity of geometry, the fluid flow has its complexities, which need a great number of grid cells to be modeled. This grid slows the solving process in order to achieve a convergent solution. In the second step, a 3-dimensional modeling is carried out. The comparison between $2 \mathrm{D}$ and $3 \mathrm{D}$ results shows the importance of third dimension in this problem.

In Lai's experiment, there are 14 ribs before test section and it is insisted that the inlet flow, before the first rib, is in fully developed turbulent regime. In Lai's experiments, the friction factor $f$, for both smooth and ribbed surface was calculated by measuring the pressure difference across two Perspex channels and can be written as:

$$
f=\frac{D_{e} / 4}{\frac{1}{2} U^{2}} \frac{\Delta P}{\Delta L}
$$

where $U\left[\mathrm{~m} . \mathrm{s}^{-1}\right]$, is the bulk mean velocity, $D_{e}[\mathrm{~m}]$, is the hydraulic mean diameter and $\Delta P / \Delta L\left[\mathrm{~Pa} . \mathrm{m}^{-1}\right]$, is the pressure loss across the length by which two Pitot tubes are separated.

For this experiment, air velocity is $4.4\left[\mathrm{~m} . \mathrm{s}^{-1}\right]$ and Reynolds number is $4.4 \times 10^{4}$. Considering Blasius equation, the friction factor for the channel is equal to:

$$
f=\frac{0.0791}{R e^{0.25}} \stackrel{\text { if } R e=1.4 \times 10^{4}}{\longrightarrow} f=5.46 \times 10^{-3}
$$

With use of friction factor, the experimental value of pressure drop per channel length can be calculated. 
This quantity is equal to $\Delta P / \Delta L=7.4437\left[\mathrm{~Pa}^{-1} \mathrm{~m}^{-1}\right]$ from experimental data. This parameter is used as a criterion to check the accuracy of converged results.

\subsection{Governing equations (for fluid)}

The air governing equation is Navier-Stokes equation for incompressible fluid without heat transfer and constant viscosity. The continuity or mass conservation equation is defined as:

$$
\frac{\partial \rho}{\partial t}+\nabla \cdot(\rho \vec{v})=0
$$

The momentum equation for inertial or non-accelerating coordinate is:

$$
\frac{\partial}{\partial t}(\rho \vec{v})+\nabla(\rho \vec{v} \vec{v})=-\nabla p+\nabla(\overline{\bar{\tau}})+\rho \vec{g}+\vec{F}
$$

In the above equation, $p$ is the static pressure, $\overline{\bar{\tau}}$ the stress tensor $[\mathrm{Pa}], \rho g$ and $F$ are gravitational and external body force, respectively.

The definition of stress tensor is as following:

$$
\overline{\bar{\tau}}=\mu\left[\left(\nabla \vec{v}+\nabla \vec{v}^{T}\right)-\frac{2}{3} \cdot \nabla \vec{v} I\right]
$$

where $\mu$ [Pa.s] is the molecular viscosity and $I$ the unit tensor.

The second term in above equation defines the volume dilatation effect.

The turbulence model which is used in this investigation is a $k-\varepsilon$ 2-equational model. Standard $k-\varepsilon$ model is a semi-experimental model based on the transport equation of turbulence kinetic energy $(k)$ and dissipation rate $(\varepsilon)$. The transport relation for $k$ is extracted from exact equation and $\varepsilon$-transport model is obtained from physical reasons which have small similarities with exact mathematical solution.

The transport equations for standard $k-\varepsilon$ model are:

$$
\begin{aligned}
\frac{\partial}{\partial t}(\rho k)-\frac{\rho}{\partial x_{i}}\left(\rho k u_{i}\right)= & \frac{\partial}{\partial x_{i}}\left[\left(\mu+\frac{\mu_{t}}{\sigma_{k}}\right) \frac{\partial k}{\partial x_{i}}\right] \\
& +G_{k}+G_{b}+\rho \varepsilon-Y_{M}+S_{k} \\
\frac{\partial}{\partial t}(\rho \varepsilon)+\frac{\partial}{\partial x_{i}}\left(\rho \varepsilon u_{i}\right)= & \frac{\partial}{\partial x_{i}}\left[\left(\mu+\frac{\mu_{t}}{\sigma_{\varepsilon}}\right) \frac{\partial \varepsilon}{\partial x_{i}}\right]+G_{1 \varepsilon} \frac{\varepsilon}{k} \\
& \times\left(G_{k}+C_{3 \varepsilon} G_{b}\right)-C_{2 \varepsilon} \rho \frac{\varepsilon^{2}}{k}+S_{\varepsilon}
\end{aligned}
$$

$G_{k}$ is the turbulence energy generation due to mean velocity gradient; $G_{b}$ the turbulence energy generation due to buoyancy; $Y_{m}$ is related to dilatation in compressible turbulence; $C_{1 \varepsilon}, C_{2 \varepsilon}, C_{3 \varepsilon}$ are constants; $\sigma_{k}, \sigma_{\varepsilon}$ are turbulence Prandtl numbers for $k$ and $\varepsilon$, respectively; $S_{k}, S_{\varepsilon}$ are source terms which can be added to equations in certain problems for $k-\varepsilon$ model; the turbulent eddy viscosity $\mu_{t}[\mathrm{~Pa} . \mathrm{s}]$ is defined as:

$$
\mu_{t}=\rho C_{\mu} \frac{k^{2}}{\varepsilon}
$$

In the above equation, $C_{\mu}$ is constant.

The constants in this model are obtained by experiments on air and water as follow:

$$
\left\{\begin{array} { l } 
{ C _ { 1 \varepsilon } = 1 . 4 4 } \\
{ C _ { 2 \varepsilon } = 1 . 9 2 } \\
{ C _ { \mu } = 0 . 0 9 }
\end{array} \text { and } \quad \left\{\begin{array}{l}
\sigma_{k}=1.0 \\
\sigma_{\varepsilon}=1.3
\end{array}\right.\right.
$$

After solving fluid turbulent flow, the micro particles are added to flow. The particle motion equation or discrete phase is studied with a Lagrangian view point. This equation which is basically the force balance for particle is written in Cartesian coordinates as below:

$$
\frac{\mathrm{d} u_{i}^{\mathrm{p}}}{\mathrm{d} t}=F_{\mathrm{D}}\left(u_{i}-u_{i}^{\mathrm{p}}\right)+\frac{g_{i}\left(\rho_{\mathrm{p}}-\rho\right)}{\rho_{\mathrm{p}}}+F_{i}^{\mathrm{L}}
$$

where $u_{i}\left[\mathrm{~m} . \mathrm{s}^{-1}\right]$ is the phase velocity, $u_{i}^{\mathrm{p}}=\mathrm{d} u_{i} / \mathrm{d} t$ $\left[\mathrm{m} . \mathrm{s}^{-1}\right]$ the particle velocity, $g_{i}$ the acceleration of gravity, $\rho$ and $\rho_{\mathrm{p}}\left[\mathrm{kg} \cdot \mathrm{m}^{-3}\right]$ are fluid and particle densities, respectively. $F_{i}^{\mathrm{L}}$ represents the components of all additional external forces acting on the particle which consider the lifting force in this case. $F_{\mathrm{D}}\left(u-u_{\mathrm{p}}\right)$ is the drag per unit particle mass, which is given by:

$$
F_{\mathrm{D}}=\frac{18 \mu}{\rho_{\mathrm{p}} d_{\mathrm{p}}^{2}} \frac{C_{\mathrm{D}} R e}{24}
$$

In the above equation, $\mu$ [Pa.s] is the fluid viscosity, and $d_{p}[\mathrm{~m}]$ the particle diameter, $R e$, the relative Reynolds number defined as:

$$
R e \equiv \frac{\rho d_{\mathrm{p}}\left|u_{i}^{\mathrm{p}}-u_{i}\right|}{\mu}
$$

For submicron particles $(100 \mathrm{~nm}-1 \mu \mathrm{m}), F_{\mathrm{D}}$ can be written as:

$$
F_{\mathrm{D}}=\frac{18 \mu}{\rho_{\mathrm{p}} d_{\mathrm{p}}^{2} C_{c}}
$$

In equation (12), $C_{c}$ is the Stokes-Cunningham slip correction factor that can be computed from:

$$
C_{c}=1+\frac{2 \lambda}{d_{\mathrm{p}}}\left(1.257+0.4 \exp -\left(\frac{1.1 d_{\mathrm{p}}}{2 \lambda}\right)\right)
$$

where $\lambda$ is the molecular mean-free path of the gas. The lifting force $F_{i}^{\mathrm{L}}$ is given by:

$$
F_{i}^{\mathrm{L}}=\frac{2 K \nu^{1 / 2} d_{i j}}{S d\left(d_{l k} d_{k l}\right)^{1 / 4}}\left(u_{j}-u_{j}^{\mathrm{P}}\right)
$$

where $K=2.594$ is the constant coefficient of Saffman lift force. The term $\left(\rho_{\mathrm{p}} / \rho\right)$ is so-called the particle-to-fluid density ratio and noted as $S \cdot d_{i j}$ is the deformation rate tensor given as

$$
d_{i j}=\frac{1}{2}\left(\frac{\partial u_{i}}{\partial x_{j}}-\frac{\partial u_{j}}{\partial x_{i}}\right)
$$




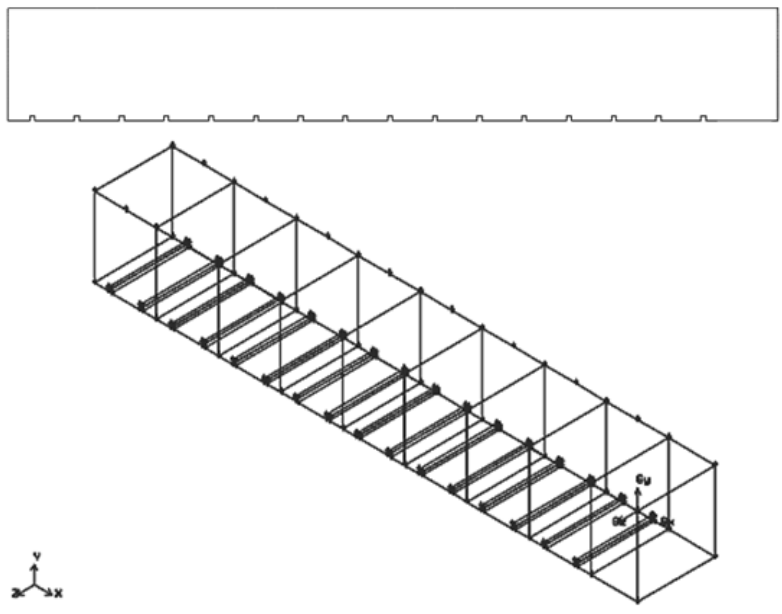

Fig. 2. Geometry of 2D and 3D models.

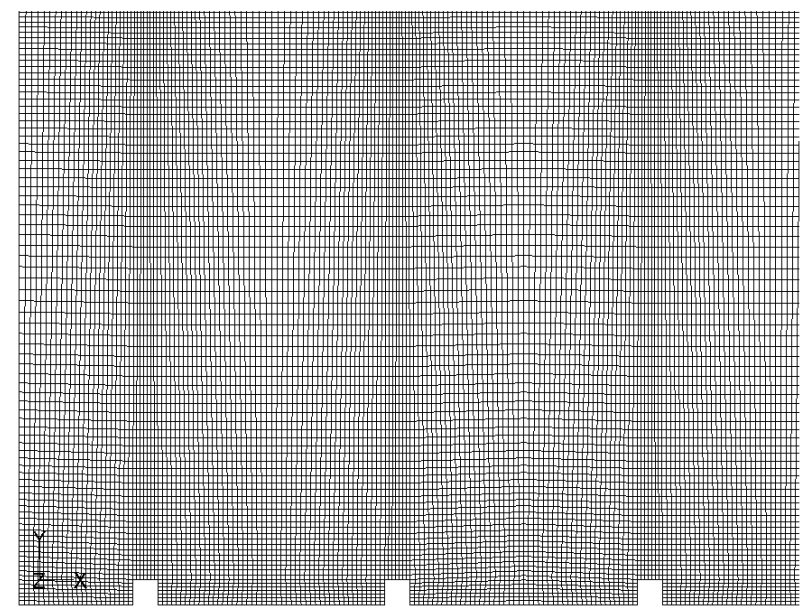

Grid FLUENT 6.3 (3d, dp, pbns, ske)

Fig. 3. Generated grid for 2D model.

\subsection{Grid generation}

For geometrical simulation two models are created separately in Gambit software. Figure 2 shows these two models.

As shown in Figure 2, at the channel end, the added zone is considered in order of avoiding recirculated flow. For the 2-dimensional model, a structured grid with 132800 elements is created. The grid quality is acceptable with a maximum equi-angle skew 0.07 and maximum aspect ratio 4.32 finally. The results grid independency is proved by comparing the results with results associated to two different grids (one with 309600 elements and other with 218016 elements). Figure 3 shows structured grid for 2D model. It is obvious that grid lines are more compressed near the walls because the flow complexities after the ribs.

The grid of 3-dimensional model is like the $2 \mathrm{D}$ model in grid quality and grid lines density. 3D grid has 3280640
3D-rectangular elements. $98.63 \%$ of elements have the acceptable aspect ratio (between 1 and 5). The maximum aspect ratio is 6 because of grid span in depth direction.

The velocity condition at inlet is defined by use of the UDF (User Defined Function). In this code the fully turbulent developed flow is defined as [12]:

$$
u=U \times\left(\frac{y}{\delta}\right)^{1 / 7}
$$

The flow is fully turbulent when $\delta$ is equal to the half of channel height. Also the equation for $k$ and $\varepsilon$ associated for fully turbulent flow is defined by this UDF. The outlet boundary condition is the pressure outlet condition.

In the following, the grid independent results for both $2 \mathrm{D}$ and $3 \mathrm{D}$ models are presented and particle deposition results are compared with experimental ones.

\section{Results}

\subsection{Fluid domain}

In the first step, the fluid domain is solved. The convergence criterion for each case is iteration independency. The outlet relative pressure for all cases is considered to be zero. According to experimental results, the pressure drop per unit length is $7.4437 \mathrm{~Pa}^{-1}$, therefore by using channel length, the pressure at the inlet boundary is determined. To obtain the inlet pressure in numerical results, the area weighted average of static pressure is calculated. Note that the outlet static pressure is zero; hence the calculated quantity is the total pressure drop in modeled channel.

It is logical to model this problem 2-dimensionally because the problem parameters do not vary considerably with depth direction. But there are main differences between $2 \mathrm{D}$ and $3 \mathrm{D}$ flows. For example, in 2D problem the effect of two lateral walls and the corner effect at inlet are neglected. As a result, it is expected that the $2 \mathrm{D}$ calculated pressure drop is less of the 3D problem result.

For the $k-\varepsilon$ turbulence model, the Enhancement Wall Treatment (E.W.T.) is chosen. This model is the best one for the $y^{+}$associated to generated grid $\left(0<y^{+}<50\right)$. For the $2 \mathrm{D}$ model, the pressure drop per unit length is $5.5329 \mathrm{~Pa} . \mathrm{m}^{-1}$ for RNG $k-\varepsilon$ model. Moreover the recirculation length after the rib is important for this problem. In the following, the velocity contours are shown for the range of stream function 0-2.0. In Figure 4 the end part of the grid or test section is drawn. From $2 \mathrm{D}$ results the length of recirculation zone after the rib is 4.52 times of rib height.

For the 3D model with standard $k$ - $\varepsilon$ turbulence model, also the Enhancement Wall Treatment is selected $(0<$ $\left.y^{+}<50\right)$. For grid independent solution, the pressure drop per unit length is $7.1566 \mathrm{~Pa}^{-1}$. The pressure contours and velocity vectors on middle plane in depth direction are shown in Figures 5 and 6 . The length of recirculation zone after the rib is 5.38 times of rib height. 


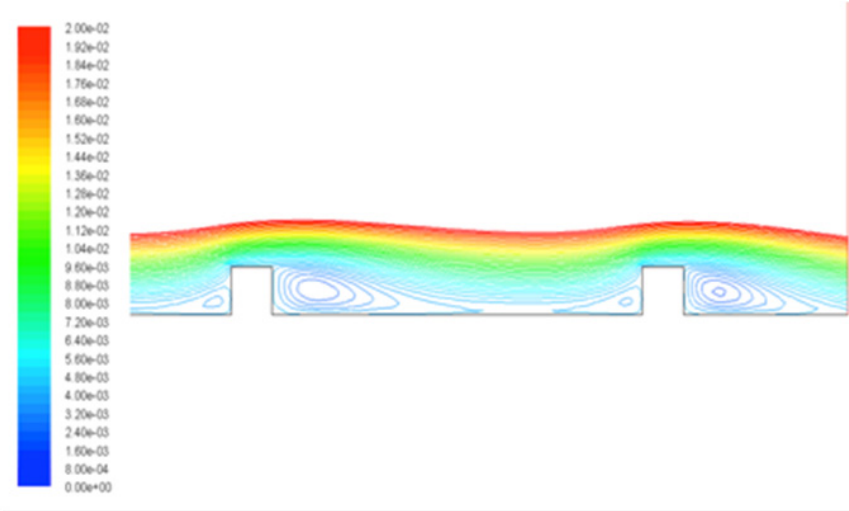

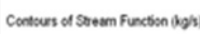
Jun 15, 2012

Fig. 4. Streamlines in test section for $2 \mathrm{D}$ model.

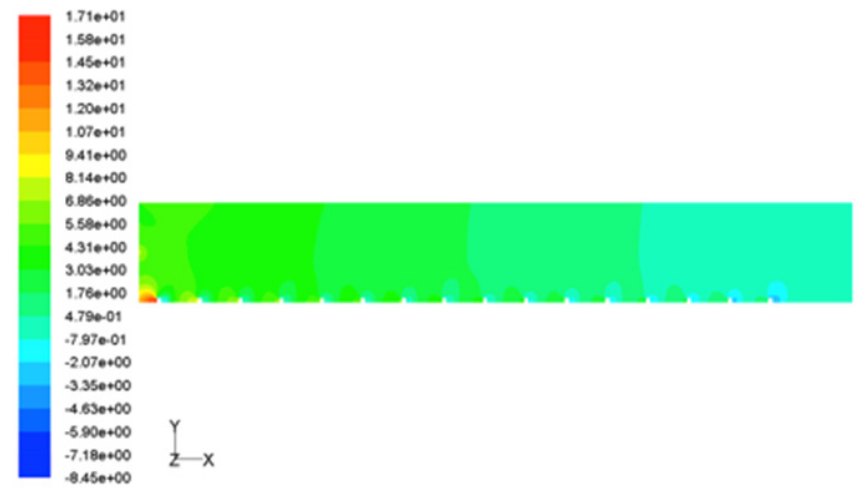

Contours of Static Pressure (pascal) Jun 17,2012
FLUENT 6.3 (3d, dp, pons, ske)

Fig. 5. Contours of static pressure on the middle surface in depth direction for 3D model.

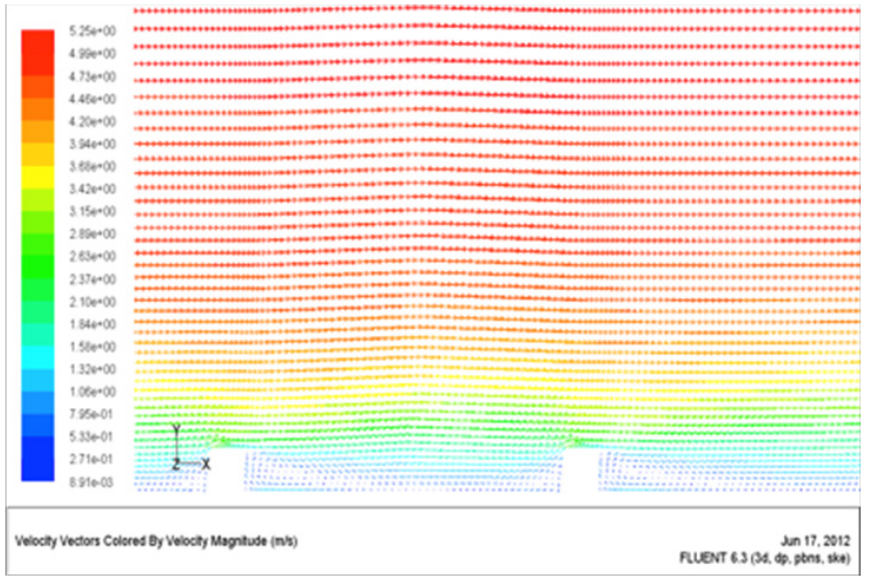

Fig. 6. Velocity vectors on the middle surface in depth direction for $3 \mathrm{D}$ model.

As it was expected, the 3D model not only calculates greater pressure drop than 2D model, but also the calculated length of recirculation zone is much greater for $3 \mathrm{D}$ model.

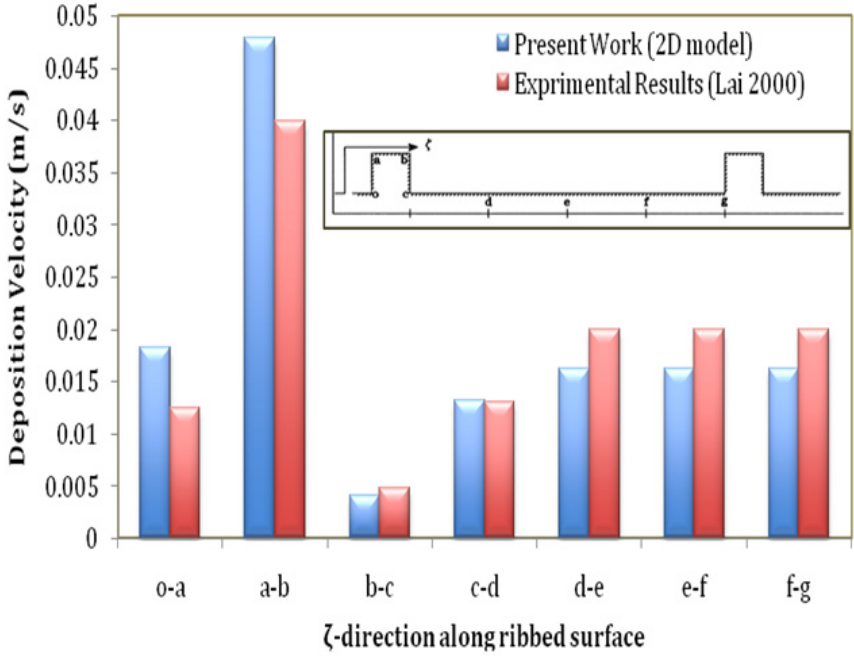

Fig. 7. Aerosol deposition velocity in ribbed channel for $2 \mathrm{D}$ model.

\subsection{Aerosol deposition}

Because the flow is turbulent, we need a model to exert the turbulence diffusion effect to the problem. In this investigation, a Stochastic Tracking model is used. The Stochastic Tracking model (Random Walk) contains the turbulence instantaneous velocity fluctuation effect on the particle trajectory by use of stochastic model. For solving particle motion in Lagrangian viewpoint, the Saffman lift force is active and Stokes-Cunningham drag law is selected.

The mass transfer coefficient (deposition velocity) $V_{\mathrm{d}}\left[\mathrm{m} \cdot \mathrm{s}^{-1}\right]$, is the key parameter to be determined. Aerosol deposition velocity is as follow [12]:

$$
V_{\mathrm{d}}=\frac{J}{C_{\infty}}
$$

In the channel, $J$ [particle.m $\mathrm{m}^{-2} \cdot \mathrm{s}^{-1}$ ] deposited aerosol flux on channel area and $C_{\infty}\left[\right.$ particle.m $\left.{ }^{-3}\right]$, aerosol concentration in free stream air, were evaluated from the following equations:

$$
\begin{aligned}
J & =\frac{M_{\text {acetate }}}{A_{\text {acetate }} t} \\
C_{\infty} & =\frac{M_{\text {filter }}}{Q}
\end{aligned}
$$

where $M_{\text {acetate }}, M_{\text {filter }}$ are the particle mass detected on the acetate and air filter paper samples respectively, $t[\mathrm{~s}]$ is the sampling time, $A_{\text {acetate }}\left[\mathrm{m}^{2}\right]$ the area of the acetate simple and $Q\left[\mathrm{~m}^{3}\right]$ is the volumetric flow rate through the air filter samplers [13].

In Figure 7, the numerical results of $2 \mathrm{D}$ models are compared with Lai's experimental results for particle with $4.5 \mu \mathrm{m}$ diameter and Figure 8 shows the same results for $3 \mathrm{D}$ model. 


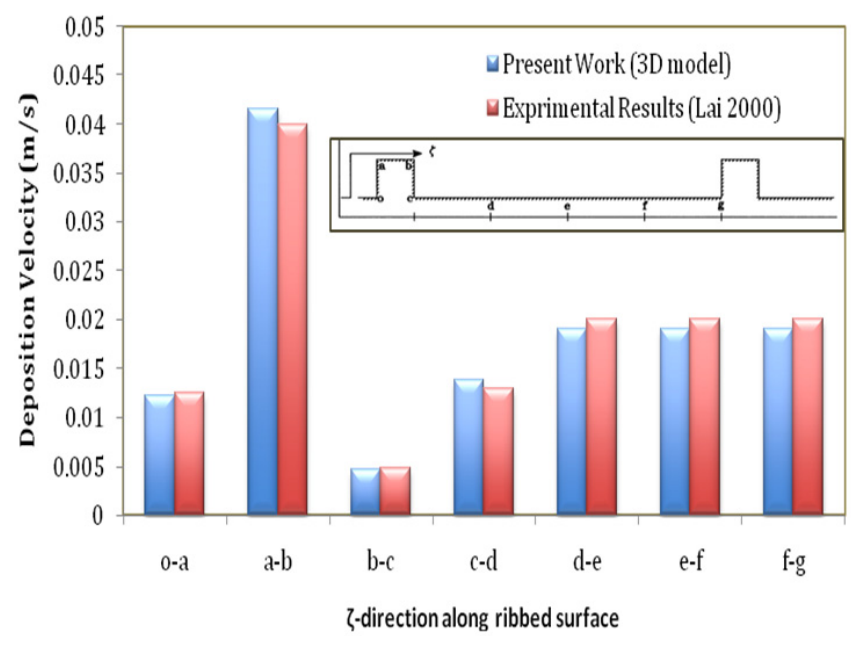

Fig. 8. Aerosol deposition velocity in ribbed channel for $3 \mathrm{D}$ model.

\section{Discussions and conclusion}

In this investigation, the importance of third dimension in numerical simulation of turbulent airflow in ribbed channel is reviewed. However the turbulence models can be exerted to $2 \mathrm{D}$ problems in commercial software, but because of 3-dimensional nature of turbulence, the 3 dimensional modeling can give better results. The difference of results for two models is $22 \%$ in the length recirculation zone after the rib. This recirculated length is measured experimentally $6 \pm 0.7$ of rib height [6]. The 3-dimensional model with a $k-\varepsilon$ turbulence model predicts this length in the range of experimental data. On the other hand the pressure drop per unit length is $7.1566 \mathrm{~Pa} . \mathrm{m}^{-1}$ for $3 \mathrm{D}$ model and $5.5329 \mathrm{~Pa}^{-1}$ for 2D model. From Lai's experimental results this parameter is $7.4437 \mathrm{~Pa} . \mathrm{m}^{-1}$. Therefore 3D model result has $3.85 \%$ error and 2D model result has $25.67 \%$ error.

Also comparing Figures 6 and 7, the 3D model has better prediction in aerosol deposition rather than $2 \mathrm{D}$ model. The greatest error in deposition velocity is $3.85 \%$ for $3 \mathrm{D}$ model and $25.67 \%$ error for 2D model.

Finally we can conclude that the $2 \mathrm{D}$ model results have greater error than $3 \mathrm{D}$ model, because the span of channel is finite. However 2D model is preferred for study the physics of flow because it needs less time to execute the calculations. With use of a suitable boundary condition the $k-\varepsilon$ model can result in accurate answers for turbulent flow in ribbed channel and also for particle deposition in these flows.

\section{References}

[1] AC.K. Lai, M.A. Byrne, A.J.H. Goddard, Aerosol deposition in turbulent channel flow on a regular array of threedimensional roughness elements, J. Aerosol. Sci. 3 (2001) $121-137$

[2] A.C.K. Lai, M.A. Byrne, A.J.H. Goddard, Enhanced particle loss in ventilation duct with ribbed surface, Build. Environ. 35 (2000) 425-432

[3] O. Labbé, J. Ryan, P. Sagaut, Direct numerical simulation of flow in ribbed channel, Int. J. Comput. Fluid Dyn. 11 (1999) 275-284

[4] W.C. Hinds, Aerosol Technology, John Wiley and Sons Inc, New York, 1999

[5] J.T. Hanley, D.S. Ensor, D.D. Smith, L.E. Sparks, Fractional Aerosol Filtration Efficiency of In-Duct Ventilation Air Cleaners, Indoor Air, pp. 169-178, 1994, Vol. 4

[6] Y. Liu, P.G. Tucker, G.L. Iacono, Comparison of zonal RANS and LES for a non-isothermal ribbed channel flow, Int. J. Heat Fluid Flow 27 (2006) 391-401

[7] A.K. Viswanathan, D.K. Tafti, Detached eddy simulation of flow and heat transfer in fully developed, Int. J. Heat Fluid Flow 27 (2006) 351-370

[8] E.A. Sewall, D.K. Tafti, A.B. Graham, K.A. Thole, Experimental validation of large eddy simulations of flow and heat transfer in a stationary ribbed duct, Int. J. Heat Fluid Flow 27 (2006) 243-258

[9] J. Ahn, H. Choi, J.S. Lee, Large eddy simulation of flow and heat transfer in a rotating ribbed channel, Int. J. Heat Mass Transfer 50 (2007) 4937-4947

[10] L. Tian, G. Ahmadi, Particle deposition in turbulent duct flows-comparisons of different model predictions, J. Aerosol. Sci. 38 (2007) 377-397

[11] J. Ahn J.S. Lee, Large eddy simulation of flow and heat transfer is a channel with a detected rib array, Int. J. Heat Mass Transfer 53 (2010) 445-452

[12] V.L. Streeter, E.B. Wylie, K.W. Bedford, Fluid Mechanics McGraw-Hill, New York, 2002

[13] A.C.K Lai, M.A. Byrne, A.J.H. Goddard, Measured deposition of aerosol particles on a two-dimentional ribbed surface in a turbulent duct flow, J. Aerosol. Sci. 30 (1999) $1201-1214$ 